\title{
Autonomous Ship Safe Navigation using Smoothing A* Algorithm
}

\author{
Yong $\mathrm{Ma}^{1,2}$, Langxiong Gan ${ }^{1}$, Yuanzhou Zheng ${ }^{1, *}$ and Jing Zhang ${ }^{3}$
}

${ }^{I}$ School of Navigation, Wuhan University of Technology; Hubei Key Laboratory of Inland Shipping Technology, Wuhan, 430063, China; ${ }^{2}$ Key Laboratory of Image Processing and Intelligent Control, Institute of Systems Engineering, Huazhong University of Science and Technology, Wuhan, 430074, China; ${ }^{3}$ School of Business, Chutian College, Huazhong Agricultural Business University, Wuhan, 430205, China

\begin{abstract}
Using reasonable and efficient autonomous technology for path planning can effectively improve the safety of ship navigation and can reduce human errors of maritime accidents. The planned path by $\mathrm{A}^{*}$ algorithm is flawed with broken lines, many turning points and numerous transitional frequencies under the environment modeled by grid method. Smoothing $A^{*}$ algorithm is adopted to delete the removable points which prolong the planned path length. After all the realities of ship navigation are taken into account, smoothing $A^{*}$ algorithm is applied to resolve safe navigation problem for the ship. With the proposed smoothing $A^{*}$ algorithm, simulation results show that the conflict-free path can be planned for ships under different circumstances with static and dynamic obstacles and abide by the COLREGS.
\end{abstract}

Keywords: Collision avoidance, COLREGS, path planning, smoothing A* algorithm, ship navigation.

\section{INTRODUCTION}

Waterway transportation occupies more than $80 \%$ of the Global trade. With the sustainable development of global trade, shipping disaster accidents take on the continued increase tendency [1]. To reduce maritime accidents evidently, and avoid the navigational risk during the process of transportation, generous solutions have been put forward to deal with those troublesome circumstances. Firstly, quantities of shipping corporations have strengthened the operation and management skills of the crew, aiming at enhancing the operations and moral quality levels of the crew. Secondly, approaches to upgrade the automatic operation ability and navigational collision avoidance level of the ship are proposed to improve the automatic operational safety levels greatly. When we come to the first aspect, lots of human factors would be involved and the mis-operation of the ship would be caused frequently. However, we cannot afford the aftereffect related to ship accidents. Consequently, to reduce the decision-making activities of human beings, it is sane and necessary to improve the automation level of the ship through scientific and technological means.

Dating from 1980s, generous researchers have paid much attention to the automatic safety navigation of the ship from the perspective of computer science and technology. To fulfill the automatic navigation for the ship, we should plan the safety and collision-avoidance paths for the ship with which the ship can navigate from its initial position to the final position. Qualities of information and many obstacles lie in the path planning problems which have been proofed as NPHard [2].
Many algorithms have been adopted to resolve path planning problems, such as the graphic method and analysis method [3]. Within the graphic method, the mostly used are the roadmap method and raster method. The path planning problem can be achieved by the roadmap method in the form of the visual diagram method or Voronoi diagram method. When comes to the raster method, the environment is divided into many grids and the search method such as Dijkstra algorithm is used to find the ideal path. Generous intelligent methods such as GA, neuron network algorithm, Ant colony algorithm, taboo search algorithm and their hybrid have been applied for path planning problems [4-7].

Little attention has been paid to the actual navigation circumstances in previous studies on safety navigation for ships. In the process of the voyage, the ship should not only avoid the obstacles but also abide by the International Regulations for preventing Collisions at Sea 1972 (COLREGS) [8]. Thus, when taken into the COLREGS in the navigational problems for the ship, some appropriate algorithm should be adopted. $A^{*}$ algorithm excels in the global path planning problems of mobile objects under the known environment, and it fits for the quadratic path planning problems for mobile objects [9]. The heuristic function has been introduced into $\mathrm{A}^{*}$ algorithm which is also called the heuristic algorithm. However, there are lots of broken lines and much turning points in the $\mathrm{A}^{*}$ algorithm and the differential $\mathrm{A}^{*}$ algorithm $[10,11]$. Therefore, to overcome above shortcomings and to integrate the COLREGS into safety navigation for the ship, we would devise the smoothing $A^{*}$ algorithm for handling these problems ideally.

\section{PROBLEM DESCRIPTION}

When it comes to the navigable area of the ship, the environment information is simplified as one two-dimensional environment. The ship is treated as one dotted moving ob- 
ject, and the obstacles such as the coastline, the reefs and the islands are mapped into the dangerous area which cannot be passed through. The sailing ship is named as $S_{\text {hip }}$ and navigates in the bounded environment $a_{\text {rea }}$, which contains numerous numbered obstacles $o_{b s i}(i=1,2, \ldots, n)$ with various shapes. $a_{r e a}$ is set to convex polygon with arbitrary shape which can be changed into the rectangular or square by way of adding certain completion area. Meanwhile, the completion area is regarded as the obstacle area. Within $a_{\text {rea }}$, its lowest left corner is presented as the origin of coordinates $O$, and the horizontal and vertical lines correspond to the $X$-axis and $Y$-axis, respectively. Consequently, after raster processing, the environmental information is mapped into the $X O Y$ coordination. Suppose the step of $S_{h i p}$ in $a_{r e a}$ is $u$, and its maximum values on the $X$-axis and $Y$-axis are $x_{\max }$ and $y_{\max }$, respectively. Thus, the number of the grids on each row denotes as $N_{c}$ equals to $x_{\max } / u$, and the number of the grids on each column denotes as $N_{l}$ equals to $y_{\max } / u$.

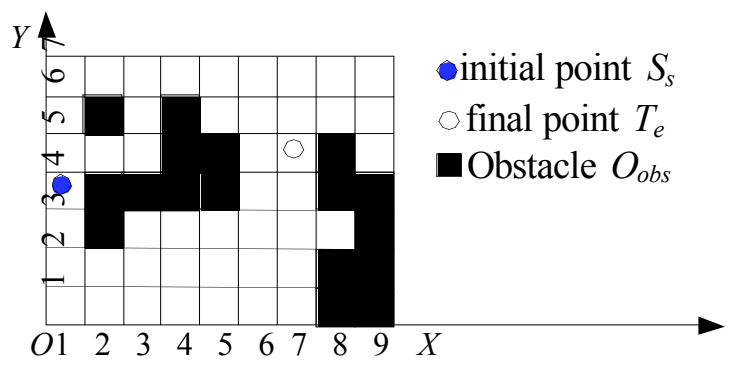

Fig. (1). Navigation environment modeled by grid method.

As shown in Fig. (1), $o_{b s i}(i=1,2, \ldots, n)$ is randomly distributed over $a_{\text {rea }}$, and the initial position of $S_{\text {hip }}$ is unfixed. $g$ represents some rand grid, and the grids of $a_{\text {rea }}$ constitute the set $A$. For $\forall o_{b s i} \in O_{o b s}(i=1,2, \ldots, n)$, the obstacle set $O_{o b s}$ which belongs to $A$ can be expressed as $\left\{o_{b s 1}, o_{b s 2}, \ldots, o_{b s n}\right\}$. Within $X O Y$, the corresponding coordinate of $g$ is $(x, y)$ and denoted as $g(x, y)$. The grid located in the lowest left corner of $X O Y$ is $(1,1)$. Suppose the set of the gird sequence number $N_{\text {num }}$ is illustrated as $\{1,2,3, \ldots, M\}$, and the $N_{\text {num }}$ of $g(1,1)$ is 1 , the $N_{\text {num }}$ of $g(2,1)$ is $\left(N_{c}+1\right)$. The coordinates of $g_{i}$ is $\left(x_{i}, y_{i}\right)$ which corresponds to the gird sequence number $i \in N_{\text {num }}\left(i \in N_{\text {num }}\right)$. To determine the coordinates of the sequence number $i$, the calculation expressions are listed as below.

$\left.\begin{array}{l}x_{i}=\left((i-1) \bmod N_{c}\right)+1 \\ y_{i}=(\operatorname{int})\left((i-1) / N_{c}\right)+1\end{array}\right\}$

After problem formulation, to resolve the safety navigation problem for $S_{\text {hip }}$ becomes a plan to optimize the paths from the initial point $S_{s}$ to the final point $T_{e}$ without colliding with any other objects, where $S, T \notin O_{o b s}, s, e \in N_{\text {num }}$, and $s \neq e$.

\section{MODEL OF SAFETY NAVIGATION FOR SHIP AND ITS FULFILMENT}

\subsection{Safety Navigation Model based on Smoothing A* Algorithm}

To dispose the safety navigation problem for the $S_{\text {hip }}$, the smoothing $A^{*}$ algorithm is devised to plan the safe and collision-avoidance path with which the $S_{\text {hip }}$ can travel from its initial point to the final point successfully. To tackle the path which has many broken lines, lots of turning points are obtained by the $A^{*}$ algorithm as shown in Fig. (2a), it is sane to process the path with some smoothing technique. Consequently, with smoothing $A^{*}$ algorithm, the path planned by A* algorithm can be processed by deleting the unnecessary connected points on the path and composing the remained points to form the newly path.

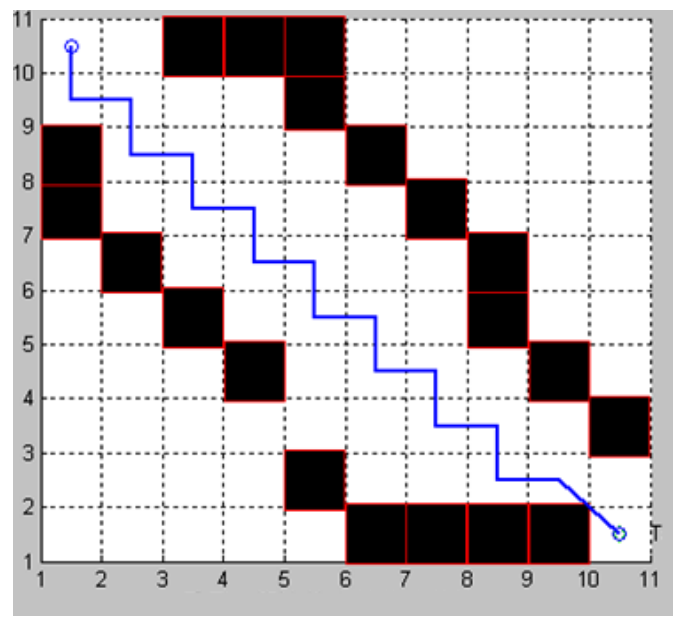

(a) Path without smoothing

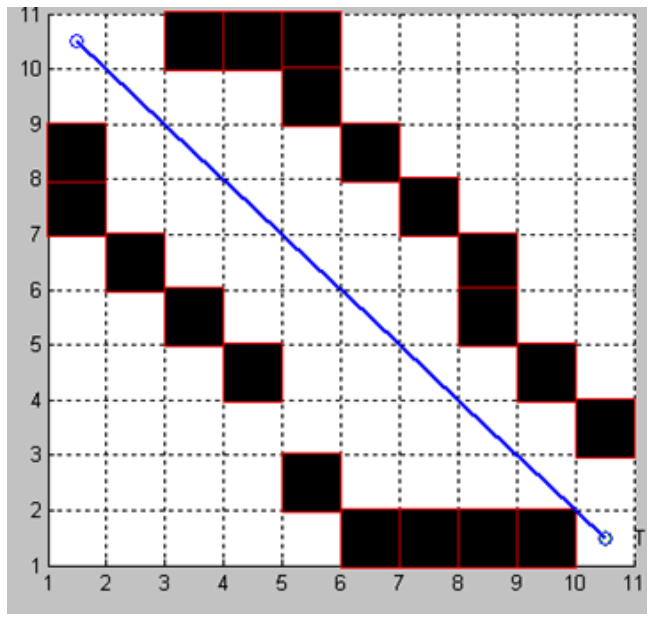

(b) Path with smoothing

Fig. (2). Planned path processed or not by smoothing algorithm.

Suppose the initial path given by $A^{*}$ algorithm named $P_{a t h 0}$, then examining all the points on the $P_{a t h 0}$ one by one, if there exists no obstacle on the line which is connected by the anterior and the immediate points of some point, then the middle point should be deleted. The flow chart of smoothing A* algorithm is elucidated in Fig. (3).

\subsection{Fulfillment of Safety Navigation Model}

To implement the model concerning the safety navigation for the ship, it requires the fulfillment of the smoothing $A^{*}$ algorithm. Thus, in the realization procession of our approach, to improve the smoothness of the planned path and reduce the accumulative turning points and turning angles, the functions named Smooth_Path ( ) and Step_able ( ) have 
been defined. The pseudocode of function Smooth_Path (GridNodes) is given below.

Algorithm Smooth Path(GridNodes)

Input: All GridNodes on the planned path

Output: The re-planned path

1 checkpoint $=\mathrm{S}_{\mathrm{s}}$

2 currentpoint $=$ checkpoint $->$ next

2 re-planned path $=$ NULL

3 while (currentpoint->next != NULL)

4 if Step_able(checkpoint, currentpoint- $>$ next)

5 temp $=$ currentpoint

6 currentpoint $=$ currentpoint $->$ next

7 delete temp from the path

8 add line between checkpoint and currentpoinit->next to re-planned path

9 else

10 checkpoint $=$ currentpoint

11 currentpoint $=$ currentpoint $->$ next

12 add line between checkpoint and currentpoinit to replanned path

13 return re-planned path

Within Step_able( ), it aims at judging if any obstacles exist or not on the line which is formed by the connected points. As shown in Fig. (2a), if we want to connect the point $S_{s}$ with the immediate point after the sequence point of $S_{S}$. We need to confirm there are no obstacles on the line connected by above two points. If obstacles exist there, then Step_able( ) returns false, which means the path is infeasible, and vice verse. The pseudocode of function Step_able( ) is given as below, where the function is used to calculate the line of two successive points.

Algorithm Step_able(checkpoint,currentpoint->next)

Input: checkpoint, currentpoint->next(both nodes on the path)

Output: false (no way), true (walkable)

1 currentpoint $=$ checkpoint $->$ next

2 line(checkpoint,currentpoint->next)

3 foreach $\left(o_{b s i}\right.$ in $\left.O b s\right)$

4 if Obsi on line

5 return false

6 else return true

Fig. (2b) shows the planned paths by our smoothing $A^{*}$ algorithm. Compared with the paths in Fig. (2a), we can find that the improved paths satisfy the path planning requirements for the safety navigation of the ship.

\section{SAFETY NAVIGATION SIMULATIONS}

\subsection{Safety Navigation Simulation Under Narrow- Channel}

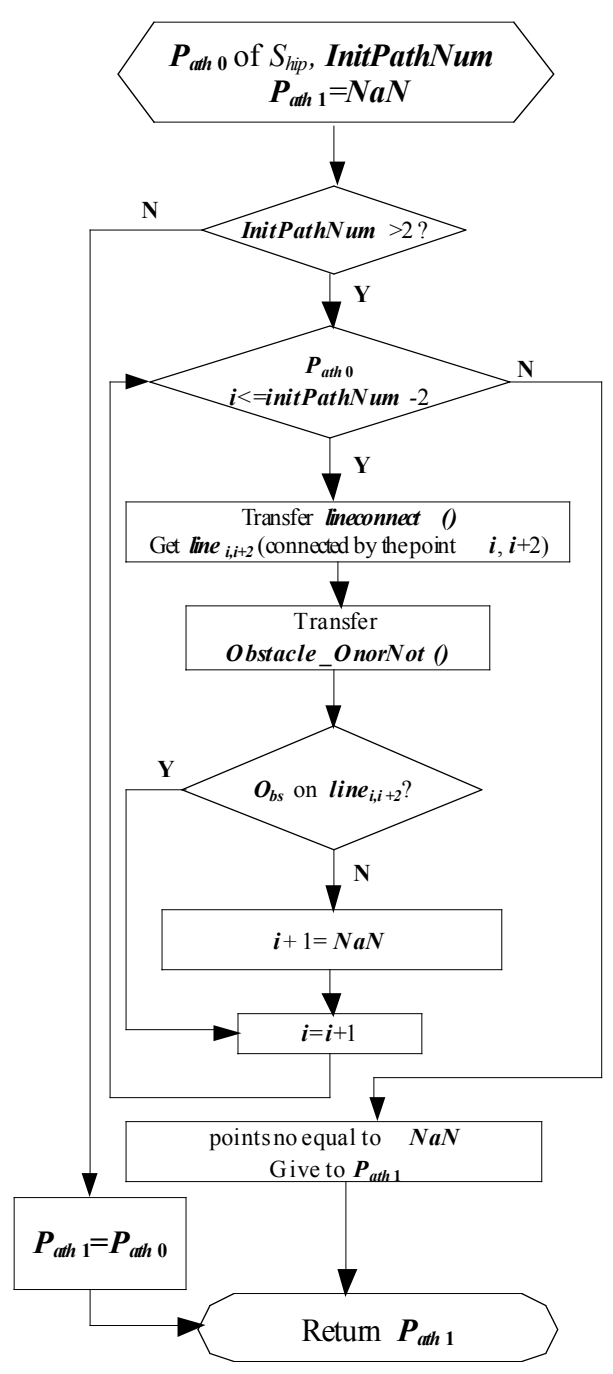

Fig. (3). Path planning flow chart for ship navigation based on smoothing $\mathrm{A}^{*}$ algorithm.

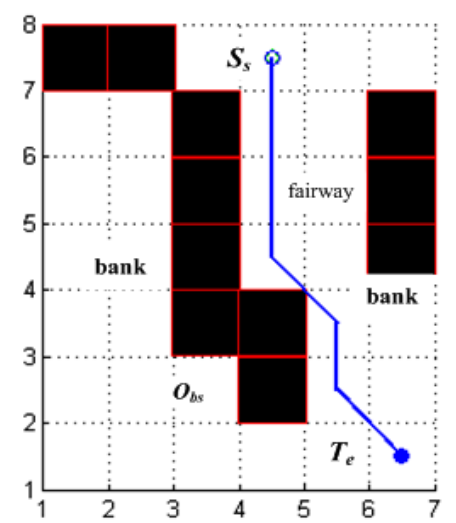

Fig. (4). Safe sailing for ship in strait channel based on smoothing A* algorithm.

As the COLREGS [8] regulated that, when sailing along the narrow-channel, the ship should be away from the obstacle and should navigate on the right side of the channel. In Fig. (4), $S_{\text {hip }}$ travels along the narrow channel, it should follow to the COLREGS and meanwhile navigate safely. Thus, the path planned by our smoothing A* algorithm is explicitly listed in Fig. (4). By using this path, the simulation shows 
that $S_{\text {hip }}$ can sail from $S_{s}$ to $T_{e}$ successfully. Simulation in Fig. (4) verifies that our smoothing $A^{*}$ algorithm fits for the safety navigation under the narrow-channel.

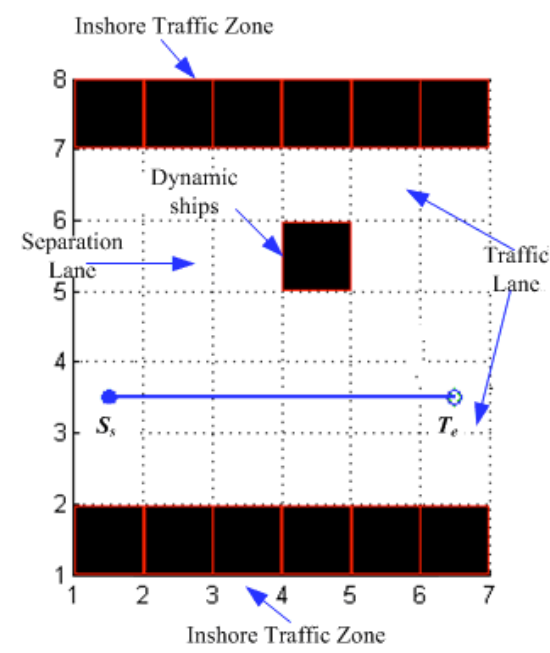

(a) Not affect by $S_{\text {hip }}$

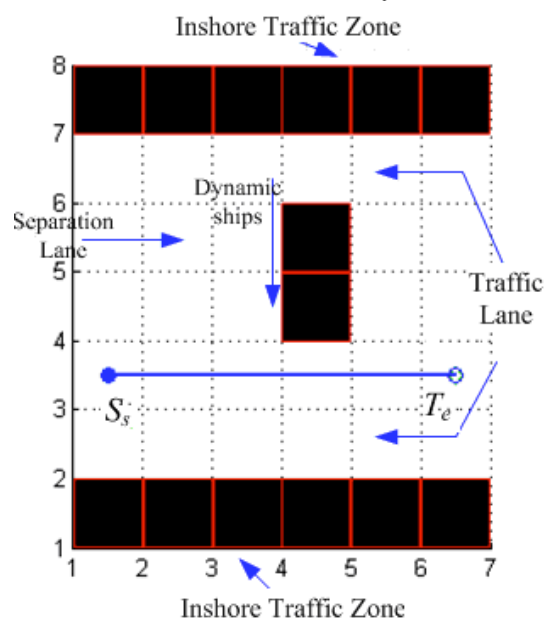

(b) Line of $S_{\text {hip }}$ affected by $S_{\text {hip }}$ Inshore Traffic Zone

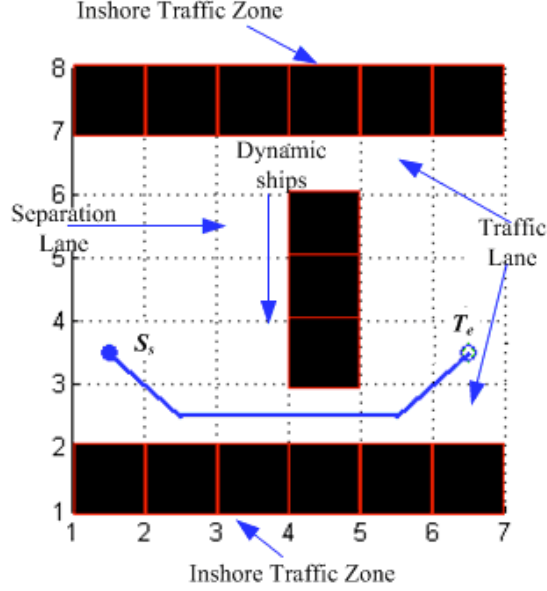

(c) lines of $S_{\text {hip }}$ affected by $S_{\text {hip }}$

Fig. (5). Safe sailing paths for ship in the restricted water area with dynamic obstacles based on smoothing A* algorithm.

\subsection{Safety Navigation Simulation Under Separate Channel}

When sailing in the separate channel, according to the good seamanship and general practice, $S_{\text {hip }}$ should navigate along the right side of the separate channel. In the process of navigating, $S_{\text {hip }}$ would excel in keeping away from the static obstacles (islands and reefs) and moving obstacles (other underway ships), and finally reach the target location.

Under the separate channel environment, generally the phenomena that lots of ships passing through the separate line would deteriorate the navigation condition of $S_{\text {hip }}$. Within separate channel as shown in Fig. (5), the inshore traffic zone is treated as an obstacle and the two traffic lanes are distributed on the right and left side of the separation lane. In Fig. (5a), the moving obstacle $O_{b s}$ has not affected the safety navigation of $S_{\text {hip }}$, and the planned path which is exhibited by the straight line is far from the traffic zone and the moving obstacles. When the dynamic ship moves, the corresponding planned path is presented as illustrated in Fig. (5b). Following that, in Fig. (5c), when the dynamic ship located on the path that is planned in Fig. (5a) and Fig. (5b), with smoothing $A^{*}$ algorithm, the planned path which is not only avoids collision but also abides by COLREGS is given in Fig. (5c).

\section{CONCLUSION}

To overcome the disadvantages of the paths planned by $\mathrm{A}^{*}$ algorithm and differential $\mathrm{A}^{*}$ algorithm, the smoothing $\mathrm{A}^{*}$ algorithm is proposed. It joins the navigational circumstances under different waterways with the COLREGS and good seamanship. The autonomous ship safe navigation problem can be resolved by our approach. Several simulations verify that the smoothing $\mathrm{A}^{*}$ algorithm can offer the effective solutions for ship safety navigation under an environment with static and dynamic obstacles.

\section{CONFLICT OF INTEREST}

The authors confirm that this article content has no conflict of interest.

\section{ACKNOWLEDGEMENTS}

This work was financially supported by National Natural Science Foundation of China (Program No. 51309186, 51479157), China Postdoctoral Science Foundation funded project (Program No. 2014M56063), Fund of Hubei Inland Shipping Technology Key Laboratory, and the fundamental research funds for the Central Universities of China (Program No. WUT: 2013-IV-105).

\section{REFERENCES}

[1] S. Saniela, and J. Rios, "Marine safety standards and the seriousness of shipping accidents," J. Nav., vol. 61, pp. 129-142, 2008

[2] D. Pratihar, and D. Kumar, "Fuzzy-genetic algorithms and timeoptimal obstacle-free path generation for mobile robots," Engin. Optim., vol. 32, pp. 117-142, 1999.

[3] J. Latombe, Robot Motion Planning. MA: Norwell, 1991, pp. 156167. 
[4] J. Barraquand, B. Langois, and J. Latombe, "Numerical potential field techniques for robot path planning," IEEE Trans. Rob. Auto. Man Cyber, vol. 22, pp. 224-241, 1992.

[5] Q. Zhu, and Y. Zhang, "An ant colony algorithm based on grid method for mobile robot path planning," Robot, vol. 27, pp. 132136, 2005.

[6] Y. Ma, H. Wang, and M. Zamirian, "A novel approach for multiple mobile objects path planning: Parametrization method and conflict resolution strategy," Physi. Lett. A, vol. 376, pp. 377-386, 2012.

[7] Y. Ma, and M. Zamirian, "Path planning for mobile objects in fourdimension based on particle swarm optimization method with penalty function," Mathe. Probl. Engin. vol. 2013, pp. 1-9, 2013.
[8] C. Cai, Paraphrase on the International Regulations for preventing Collisions at Sea 1972 (COLREGS). Beijing, 1995, pp. 20-46.

[9] P. Hart, N. Nilsson, and B. Raphael, "A formal basis for the heuristic determination of minimum cost paths," IEEE Trans. Syst. Sci. Cyber, vol. 4, pp. 100-107, 1968.

[10] K. Trovato, and L. Dorst, "Differential A*," IEEE Trans. Knowl. Data Eng, vol. 14, pp. 1218-1229, 2002.

[11] H. Wang, Y. Ma, and Y. Xie, "Mobile robot optimal path planning based on smoothing A* algorithm," J. Tongji Univ., vol.38, pp. $1647-1655,2010$.

(C) Ma et al.; Licensee Bentham Open.

This is an open access article licensed under the terms of the Creative Commons Attribution Non-Commercial License (http://creativecommons.org/licenses/by-nc/3.0/) which permits unrestricted, non-commercial use, distribution and reproduction in any medium, provided the work is properly cited. 